\title{
A GENERALIZATION OF THE ALMOST ZERO THEORY
}

\author{
R. SRIDHARAN *
}

\section{Introduction}

In this note we shall give a modified definition of cohomology groups for algebras. For a class of (infinite rank) algebras (which includes Frobenius algebras, group rings of infinite groups and division algebras of countable rank over fields), these groups can be characterized in a manner similar to the cohomology groups in the 'Almost Zero theory' of B. Eckmann [2]. Actually, in the case of group rings, these coincide with the cohomology groups in the almost zero theory.

We shall see that a theorem [3, Th. 11] of S. Eilenberg and T. Nakayama can be deduced from our general setting. We shall also derive a necessary and sufficient condition for modules over algebras of the above class to be weakly projective, which is a generalization of a proposition $[1$, p. 200] of $\mathrm{H}$. Cartan and S. Eilenberg on modules over group rings. For all notions relating to Homological algebra, we refer to [1].

\section{The almost zero theory}

We shall recall here the definition of the almost-zero theory $[1$, p. 358]. Let $\pi$ be a group, $C$ an abelian group with trivial $\pi$-operators. Let $X$ be a left $\pi$-complex. $A$ cochain $f \in \operatorname{Hom}_{Z}\left(X_{n}, C\right)$ is called a $\pi$-finite cochain if for any $p \in X_{n}, f(x \cdot p)=0$ for all but a finite number of $x \in \pi$. For any left $Z(\pi)$-module $A$, we have an isomorphism $[1, \mathrm{p} .359]$

$$
\operatorname{Hom}_{Z(\pi)}(A, Z(\pi) \underset{Z}{\otimes}, C) \approx \overline{\operatorname{Hom}}_{Z}(A, C)
$$

where $\operatorname{Hom}_{Z}(A, C)$ is the subgroup of $\operatorname{Hom}_{Z}(A, C)$ consisting of all homomor-

Received August 1, 1958.

* The author is extremely indebted to Professor K. Chandrasekharan for his constant advice and encouragement. The author is also thankful to Mr. N. Ramabhadran for his helpful suggestions in the exposition of the text. 
phisms $f$ which satisfy for every fixed $a \in A$, the condition $f(x \cdot a)=0$ for all but a finite number of $x \in \pi$. Replacing $A$ by a projective resolution of $Z$ as a left $Z(\pi)$-module, and passing to homology, we have

$$
H^{n}\left(\pi, Z(\pi) \otimes{ }_{z} C\right) \approx H^{n}\left(\overline{\operatorname{Hom}}_{z}(X, C)\right) .
$$

The groups $\mathrm{H}^{n}(\pi, C)=H^{n}\left(\overline{\mathrm{Hom}}_{z}(X, C)\right)$ are the cohomology groups in the almost zero theory.

Thus the above isomorphism relates the almost zero theory with the usual cohomology theory.

\section{The groups $\widetilde{H}^{n}(\Lambda, C)$}

We shall now define, for any algebra $A$ (always assumed to be with unit element) over a commutative ring $K$ (also with unit element) the groups $\widetilde{H}^{n}(A, C)$ by

$$
\tilde{H}^{n}(\Lambda, C)=H^{n}\left(\Lambda, A \otimes \otimes_{\mathrm{K}} C\right)
$$

where $C$ is a right $A$-module.

Remark. In the case of group rings, or more generally, in the case of supplemented algebras, it is enough if $C$ is $K$-module, since we can define the right $A$-module structure, by using supplementation. But in the case of arbitrary algebras, it is not in general true that a $K$-module without 1 -operators can be considered in a natural way as a A-module. Thus, we assume $C$ is a right $A$-module and get a two-sided $d$-module by taking the "covariant extension".

Definition. Let $\check{\operatorname{dim}} A=$ greatest integer $n$ (if it exists) such that $\widetilde{H}^{n}(\Lambda, C)$ $\neq(0)$ for at least one $C_{\Lambda}$. If no such integer exists, let $\widetilde{\operatorname{dim}} \Lambda=\infty$.

Proposition 1.

$$
\widetilde{\operatorname{dim}} \Lambda=\operatorname{dim} \Lambda \text { if } \operatorname{dim} A<\infty .
$$

Proof: From our definitions, it follows that $\widetilde{\operatorname{dim}} \Lambda \leq \operatorname{dim} A$. Suppose on the other hand $\operatorname{dim} A=n<\infty$. Then there exists a $A^{e}$-module $A$ such that $H^{n}(A, A) \neq(0)$ and $H^{n+1}(A, B)=(0)$ for any $A^{e}$-module $B$. Consider now the exact sequence

$$
0 \longrightarrow R \longrightarrow \Lambda \underset{K}{\otimes} A \stackrel{g}{\longrightarrow} A \stackrel{\leftrightarrow}{\longrightarrow} 0
$$


where $g(\lambda \otimes a)=\dot{\lambda} a$, and $R=\operatorname{ker} g$.

This is easily seen to be a $1^{e}$-exact sequence. Writing down the exact cohomology sequence, we get an epimorphism $H^{n}(A, A \otimes A) \rightarrow H^{n}(.1, A)$ and since $H^{n}(\Lambda, A) \neq(0), H^{n}(.1,1 \otimes A) \neq(0)$, hence $\widetilde{\operatorname{dim}} .1 \doteq n$.

Proposition 2. Let 1 be a Frobenius algebra [3, p. 3] over a commutative ring $\mathrm{K}$. Then $\widehat{\operatorname{dim}} A=0$.

Proof: Since 1 is a Frobenius algebra, there is a natural left 1 -isomorphism $1 \underset{K}{\infty} C \approx \operatorname{Hom}_{K}(A, C)$ and because of the naturality of this mapping, it is also a right $A$-isomorphism (since $C$ has a right $A$-module structure). Thus there is a $A^{e}$-isomorphism $A \underset{K}{\times} C \approx \operatorname{Hom}_{K}(A, C)$. But $H^{n}\left(A, \operatorname{Hom}_{K}(A, C)\right.$ ) $=(0)$ if $n>0$ (See for instance, [4, p. 74]). Hence the required result.

Remark. Propositions 1 and 2 imply that if $A$ is a Frobenius algebra over a commutative ring $K, \operatorname{dim} A=0$ or $\infty$, a theorem [3, Th. 11] of S. Eilenberg and T. Nakayama.

\section{A characterization of $\widetilde{H}^{n}(\Lambda, C)$ for a class $t 3$ of algebras}

We shall now give a characterization of these groups $\tilde{H}^{n}(1, C)$ for a class of algebras of infinite rank and prove that it coincides with the almost zero cohomology groups of B. Eckmann.

Let 1 be a $K$-free algebra (not necessarily of finite rank) over an integral domain $K$. Let us assume further that there exists a non-degenerate associative bilinear form $B$ on $A \times A$, i.e.

$$
\begin{aligned}
& B(\lambda, \mu)=0 \text { for every } \mu \Rightarrow \lambda=0 \\
& B(\lambda, \mu)=0 \text { for every } \lambda \Rightarrow \mu=0 \\
& B(\lambda, \mu \nu)=B(\lambda \mu, \nu)
\end{aligned}
$$

Let us denote $B(\lambda, \mu)$ by $\langle\lambda, \mu\rangle$.

Let $\left(\lambda_{i}\right)_{i \in I}$ be a $K$-basis of $A$. Let us assume that there exists a "dual basis" $\left(\bar{\lambda}_{i}\right)_{i \in I}$ with respect to $B$. The class $\&$ by definition consists of all such algebras. We shall see later that this class covers quite a number of algebras.

The bilinear form $B$ induces a 1 -monomorphism

$$
\begin{aligned}
& \phi: A \rightarrow 1^{\circ}(\text { the dual of } \lambda) \text { defined by } \\
& \phi(\lambda)(\mu)=B(\mu, \lambda)
\end{aligned}
$$


(The fact that $\phi$ is a monomorphism follows from the non-degeneracy of $B$ and that it is 1 -homomorphism follows from the associativity of $B$ ).

There is an induced .1-homomorphism

$$
\phi \otimes I_{C}: A \underset{K}{\otimes} C \rightarrow 1^{\circ} \underset{\kappa}{\otimes} C\left(I_{C} \text { denoting the identity map of } C\right. \text { ) }
$$

and a homomophism

$$
\psi: A^{\circ} \underset{K}{\otimes} C \rightarrow \operatorname{Hom}_{K}(A, C)
$$

defined by $\psi(f \otimes c)(\lambda)=f(\lambda) \cdot c, f \in 1^{\circ}, c \in C$. By the naturality of $\phi$, it follows that $\psi$ is a 1 -homomorphism. Composing, we get a $A$-homomorphism

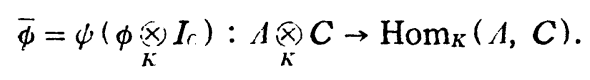

Proposition 3. $\bar{\phi}$ is a A-monomorphism.

Suppose

$$
\bar{\phi}\left(\sum_{i=1}^{n} \lambda_{i} \otimes c_{i}\right)(\not l)=\sum_{i=1}^{n}\left\langle\mu, \lambda_{i}\right\rangle c_{i}=0
$$

for every $\iota \in A$. Take $\iota=\bar{\lambda}_{j}, j=1, \ldots, n$. We get $c_{j}=0, j=1, \ldots, n$. Thus $\sum_{i=1}^{n} \lambda_{i} \otimes c_{i}=0$

Let $A$ be a left $A$-module. Since Hom $_{\Lambda}$ is left exact, there is a monomorphism

$$
\operatorname{Hom}_{\Lambda}(A, A \underset{K}{\otimes}) \rightarrow \operatorname{Hom}_{\Lambda}\left(A, \operatorname{Hom}_{K}(A, C)\right) .
$$

The right hand side of the above is naturally isomorphic with $\operatorname{Hom}_{K}(A, C)$ by the associativity formula. Thus we get a monomorphism

$$
i: \operatorname{Hom}_{\Lambda}(A, A \underset{K}{\otimes} C) \rightarrow \operatorname{Hom}_{K}(A, C),\left({ }_{\Lambda} A,{ }_{K} C\right)
$$

Let $\widehat{\operatorname{Hom}}_{K}(A, C)$ denote the image of $i$.

Proposition 4. $\widehat{\operatorname{Hom}}_{K}(A, C)$ consists precisely of those maps $f$ which satisfy the "finiteness" condition that for any fixed $a \in A, f\left(\bar{\lambda}_{i} \cdot a\right)=0$ for all but a finite number of $\left(\bar{\lambda}_{i}\right)$.

Proof: Let $f \in \widetilde{\operatorname{Hom}}_{K^{-}}(A, C), f=i(g)$ and let

$$
g(a)=\sum_{i=1}^{n} \lambda_{i} \otimes c_{i}, \quad a \in A
$$


Then

$$
\begin{aligned}
f(a) & =\sum_{i=1}^{n}\left\langle 1, \lambda_{l}\right\rangle c_{i} \\
f\left(\bar{\lambda}_{j} \cdot a\right) & =\sum_{i=1}^{n}\left\langle\bar{\lambda}_{j}, \lambda_{i}\right\rangle c_{i} \\
& =c_{j} .
\end{aligned}
$$

Thus $f\left(\vec{\lambda}_{j} \cdot a\right)=0$ for $j \neq 1,2, \ldots, n$. Conversely, if $f$ is such that $f\left(\bar{\lambda}_{i} \cdot a\right)=$ 0 for all but a finite number of $\left(\bar{\lambda}_{i}\right)$ say $\bar{\lambda}_{1}, \ldots, \bar{\lambda}_{n}$ define the map $g$ by

$$
g(a)=\sum_{i=1}^{n} \lambda_{i} \otimes f\left(\bar{\lambda}_{i} \cdot a\right)
$$

$g$ is a $A$-homomorphism since $B$ is associative, and

$$
i g\left(\bar{\lambda}_{i} \cdot a\right)=f\left(\bar{\lambda}_{i} \cdot a\right) .
$$

Since $\left(\bar{\lambda}_{i}\right)$ is a basis, $\operatorname{ig}(a)=f(a)$ for every $a \in A$.

Remark 1. In the case $A=Z(\pi)$ where $\pi$ is a group, we can take the elements of $\pi$ as a base for $A$ and the bilinear form is defined by $(x, y)$ $=\delta_{x, y^{-1}} x, y \in \pi$ and then extended to $Z(\pi)$ by bilinearity. The dual base in this case coinides with $\pi$ itself. Thus $A=Z(\pi) \in \nVdash$ and the above proposition shows that the image $\widetilde{\operatorname{Hom}}_{Z}(A, C)$ in this case is the subgroup of all additive maps $f$ which satisfy for any fixed $a \in A$, the condition $f(x \cdot a)=0$ for all but a finite number of $x \in \pi$. That is $\widetilde{\operatorname{Hom}}_{Z}(A, C)=\widetilde{\operatorname{Hom}}_{Z}(A, C)$ (defined in the beginning of the note). If we replace $A$ by a projective resolution of $Z$ as a $Z(\pi)$-module, and pass to homology, we get the cohomology groups in the almost zero theory.

Let $C$ be a right $A$-module. Then $A \underset{K}{\otimes} C$ is a $A^{e}$-module. Consider the composite $i^{\prime \prime}=i i^{\prime}$.

$$
\operatorname{Hom}_{\Lambda^{\prime \prime}}(A, A \underset{K}{\otimes} C) \stackrel{{ }^{\prime}}{\longrightarrow} \operatorname{Hom}_{\Lambda}(A, A \underset{h}{\otimes} C) \stackrel{i}{\longrightarrow} \operatorname{Hom}_{K}(A, C) .
$$

The first is a monomorphism, since every $1^{e}$-homomorphism is a 1 -homomorphism. Since the second is also a monomorphism, the composite is a monomorphism.

Propositron 5. The image of $i^{\prime \prime}$ is the set of all right 1-homomorphisms $f: A \rightarrow C$ which satisfy for any fixed $a \in A$, the condition that $f\left(\bar{\lambda}_{i} \cdot a\right)=0$ for all but a finite number of $\bar{\lambda}_{i}$. 
Proof: Let $g \in \operatorname{Hom}_{\Lambda^{c}}(A, \alpha \otimes C)$ and $g(a)=\sum_{i=1}^{13} \lambda_{i} \otimes c_{i}$

$$
\begin{aligned}
i^{\prime \prime}(g)(a \cdot \lambda) & =\sum_{i=1}^{n}\left\langle 1, \lambda_{i}\right\rangle \cdot c_{i} \cdot \lambda \\
& =\left(\ell^{\prime \prime}(g)(a)\right) \lambda .
\end{aligned}
$$

Hence $i^{\prime \prime}(g) \in \operatorname{Hom}_{\Lambda}(A, C)$ (the $K$-module of right $A$ homomorphisms $A \rightarrow C$ ). We have already seen, in Proposition 4, $i^{\prime \prime}(g) \in \widetilde{H o m}_{K}(A, C)$. Conversely, if $f \in \operatorname{Hom}_{\Lambda}(A, C) \cap \widetilde{\operatorname{Hom}}_{K}(A, C)$, then the corresponding $g$ is given by

Then

$$
\begin{aligned}
g(a) & =\sum \lambda_{i} \otimes f\left(\bar{\lambda}_{i} \cdot a\right) \\
g(a \cdot \lambda) & =\sum \lambda_{i} \otimes f\left(\bar{\lambda}_{i} a \cdot \lambda\right) \\
& =\sum \lambda_{i} \otimes f\left(\lambda_{i} \cdot a\right) \cdot \lambda \\
& =g(a) \cdot \lambda .
\end{aligned}
$$

Thus $g \in \operatorname{Hom}_{\Lambda^{e}}(A, \Lambda \underset{K}{\otimes} C)$. Let $\widetilde{\operatorname{Hom}_{\Lambda}}(A, C)=\operatorname{Hom}_{\Lambda}(A, C) \cap \widetilde{\operatorname{Hom}_{K}}(A, C)$. We have then an isomorphism

$$
\operatorname{Hom}_{\Lambda^{e}}\left(A, \Lambda \otimes_{K} C\right) \approx \overbrace{\operatorname{Hom}_{\Lambda}}(A, C) \quad\left({ }_{\Lambda^{e}} A, C_{\Lambda}\right)
$$

Replacing $A$ by a $\Lambda^{e}$-projective resolution $X$ of $\Lambda$, we get

$$
\tilde{H}^{n}(\Lambda, C)=H^{n}(\Lambda, \Lambda \otimes C) \approx H^{n}\left(\widetilde{\operatorname{Hom}}_{\Lambda}(X, C)\right) .
$$

This gives a characterization of the groups $\widetilde{H}^{n}$ which is similar to that of the almost zero theory.

We shall now prove that the groups $\widetilde{H}^{n}$ are isomorphic to the $\bar{H}^{n}$ occurring in the almost zero theory of groups. More generally, let $\Lambda$ be a supplemented $K$-algebra with supplementaion $\varepsilon$. Let $C$ be a $K$-module without $A$. operators. Let $C_{\varepsilon}$ denote the module $C$ with trivial right $A$-operators. $A \underset{K}{\otimes} C_{\varepsilon}$ is a $A^{e}$-module. On the other hand, consider $A \underset{K}{\otimes} C$. It is a left $A$-module and has no right $A$-operators. Consider $(A \otimes C)_{\varepsilon}$. Then it is seen that the $A^{e}$. modules $A \otimes C_{\varepsilon}$ and $(A \otimes C)_{\varepsilon}$ are $A^{e}$-isomorphic. We now state the

Proposition 6.

$$
\widetilde{H}^{n}\left(A, C_{\varepsilon}\right) \approx \operatorname{Ext}_{\Lambda}{ }_{\Lambda}(K, \underset{K}{\otimes} C){ }_{K} C_{K}
$$

Proof: By a theorem [1, p. 185] of H. Cartan and S. Eilenberg, it follows that

$$
\left.\operatorname{Ext}_{\Lambda}^{n}(K, \Lambda \underset{\kappa}{\otimes} C) \approx H^{n}(\Lambda, \underset{K}{(} \underset{\kappa}{\otimes} C)_{\varepsilon}\right)
$$


By the preceding remarks, it follows that the right hand side of the above isomorphism is again isomorphic to $H^{n}\left(A, A \underset{K}{\otimes} C_{\varepsilon}\right)$. But $H^{n}\left(A, A \otimes C_{\xi}\right)=\tilde{H}^{n}$ $\left(A, \boldsymbol{C}_{\varepsilon}\right)$.

Specialising $A$ to be group ring $Z(\pi)$ of a group $\pi$ over $Z$, we get that the $\tilde{H}^{n}$ are isomorphic to the $\bar{H}^{n}$.

\section{Examples of algebras of the class $\odot$}

We have assumed so far that our algebras $A$ belong to the class $€$. We shall now give examples of such algebras.

Example 1. Let $\pi$ be a group and $K(\pi)$ the group ring of $\pi$ over an integral domain $K$ with unit element. This leads, as we have seen already to, the Almost Zero theory of B. Eckmann.

Example 2. Let $A$ be a $K$-free Frobenius algebra over an integral domin $K$. (This includes group rings of finite groups, semi-simple algebras of finite rank over a field, matrix algebras, etc.). The proof of the existence of dual basis is well known.

Example 3. Let $A$ be a division algebra (with unit element) of countable rank over a field $K$. Then any non-zero linear map $\Lambda \rightarrow K$ gives rise to an associative, non-degenerate bilinear form. The existence of dual basis to a given basis is guaranteed by a more general proposition of G. W. Mackey on vector spaces in duality. [See for instance, 6, p. 88].

We shall give a condition for modules over algebras of class $€$ to be weakly projective which is a generalization of a proposition [1, p. 200] of H. CartanS. Eilenberg.

Proposition 7. Let 1 be an algebra of class ß.. A necessary and sufficient condition that a left A-module $A$ be weakly projective is that there exists $a \mathrm{~K}$ endomorphism $f: A \rightarrow A$ such that for each $a \in A$

(1) $\quad f\left(\bar{\lambda}_{i} \cdot a\right)=0$ for all but a finite number of $\bar{\lambda}_{i}$.

(2) $\quad a=\sum_{i \in I} \lambda_{i} f\left(\bar{\lambda}_{i} \cdot a\right)$.

Remark. The corresponding proposition of H. Cartan and S. Eilenberg uses very strongly the group properties. Our proof is more general and applies to 
all algebras of class $\overparen{C}$.

Proof: Suppose such a mapping exists. Define a mapping

$$
\begin{gathered}
f^{\prime}: A \rightarrow \Lambda \underset{K}{\otimes} A \text { by } \\
f^{\prime}(a)=\sum_{i \in I} \lambda_{i} \otimes f\left(\bar{\lambda}_{i} a\right)
\end{gathered}
$$

Since the bilinear form is associative, $f^{\prime}$ is a $A$-homomorphism. (2) implies that the composite $A \stackrel{f^{\prime}}{\longrightarrow} A \otimes A \longrightarrow A$ is identity. Since $1 \underset{\kappa}{\otimes} A$ is weakly projective [1, p. 197], $A$ is weakly projective. Suppose, conversely, that $A$ is weakly projective. Then there exists a $A$-homomorphism $f^{\prime}: A \rightarrow \Lambda \underset{K}{\otimes} A$ such that the composite $A \stackrel{f^{\prime}}{\longrightarrow} A \underset{K}{\otimes} A \longrightarrow A$ is identity. Consider the image $f$ of $f^{\prime}$ in $\operatorname{Hom}_{K}(A, A)$ under the monomorphism

$$
\operatorname{Hom}_{\Lambda}(A, A \underset{K}{\otimes} A) \rightarrow \operatorname{Hom}_{K}(A, A)
$$

defined before. Then, by Proposition 4, for any fixed $a \in A, f\left(\bar{\lambda}_{i} \cdot a\right)=0$ for all but a finite number of $\bar{\lambda}_{i}$. Since $f^{\prime}(a)=\sum \lambda_{i} \otimes f\left(\bar{\lambda}_{i} \cdot a\right)$, it follows that $\sum \lambda_{i} f\left(\bar{\lambda}_{i} \cdot a\right)=a$. Hence the proposition.

Remarks: (1) We have considered so far only the cohomology of algebras. A similar treatment can be given for homology. We difine first

$$
\tilde{H}_{n}(\Lambda, C)=H_{n}\left(\Lambda, \operatorname{Hom}_{K}(\Lambda, C)\right){ }_{\Lambda} C
$$

and we can prove propositions analogous to the ones we have in the case of cohomology. But we do not go into the details.

(2) The results can be relativized using the notions of the relative homological algebra of G. Hochschild [5]. Here we will have to consider a pair ( $A, \Gamma$ ) of a ring $A$ and a subring $\Gamma$ (not necessarily commutative).

\section{REFERENCES}

[1] H. Cartan and S. Eilenberg, Homological algebra, Princeton, 1956.

[2] B. Eckmann, On complexes over a ring and restricted cohomology groups, Proc. Nat. Acad. Sci. 33 (1947), 275-281.

[3] S. Eilenberg and T. Nakayama, On the dimension of modules and algebras II, Nagoya Math. Jour., 9 (1955), 1-16.

[4] S. Eilenberg, A. Rosenberg and D. Zelinsky, On the dimension of modules and algebras VIII, Nagoya Math. Jour., 12 (1957), 71-93. 
[5] G. Hochschild, Relative Homoloğical Algebra, Trans. Amer. Math. Soc., 82 (1956), 246269.

[6] N. Jacobson, Stucture of Rings, A.M.S. Colloq. publns.,(1956).

Tata Institute of Fundamental Research

Bombay 1 